\title{
Coherence Effects in Electron Diffraction from Presolar Graphenes
}

\author{
E. Mandell, P. Fraundorf \\ Department of Physics and Astronomy and Center for Molecular Electronics \\ University of Missouri - St. Louis, St. Louis, Missouri 63121
}

This set of analyses examines how coherence effects for coordinated atom-thick structures evidence themselves in diffraction. It also provides an example where this effect may be occurring in nature, through the examination of carbonaceous materials condensed from meteorites. For atom-thick structures, these coherence effects may be seen in diffraction, and they provide additional structural information beyond simple lattice periodicities.

The target here is unlayered graphene in the core of a subset of the isotopically heavy (i.e. with ${ }^{12} \mathrm{C} /{ }^{13} \mathrm{C}<$ solar=89), micron-sized, ultramicrotomed graphite spherules from the Murchison meteorite (Fig. 1) [1]. These presolar particles are characterized by "graphite onion" rims (with a concentric arrangement of $0.34[\mathrm{~nm}]$ (002) graphite layers) surrounding the spherical cores of interest. The core is comprised primarily of atom-thick graphene "flakes" [2, 3], as shown e.g. by the presence of graphite (hk0) ordering in electron diffraction, with no sign of the $0.34[\mathrm{~nm}](002)$ graphitic layering characteristic of graphite, amorphous carbon, multi-wall carbon nanotubes, and most solid non-diamond carbon phases (Fig. 1).

While flat-sheet diffraction models for graphene sheets fit experimental data from the cores reasonably well, systematic differences arise. For example, we have applied Warren models [4] for single-sized flat sheets, two sheet sizes, and a log-normal size distribution of flat sheet sizes to fit the experimental data. Each of these left the extra scattering at the graphene peaks and on the leading (low-frequency) edge unresolved. Also, the same residual effects were seen in experimental diffraction patterns from different spherules. One explanation for these differences is the coherence effect that results from coordination between adjacent graphene sheets. Fig. 2 contains a model faceted nanocone and the three dimensional reciprocal lattice structure corresponding to the (110) lattice spacing for each sheet. Of interest are the intersections of the harmonics, where the triple intersections serve to slightly broaden or shift the location of the (110) peak, and where the double intersections (where two rel-rods nearly touch) result in a satellite peak. For clarity, Figure 3 shows a modeled azimuthally-averaged diffraction profile for two adjacent facets and a single facet, or flat sheet. If the angle between the facets is changed, these coherence spikes will also move, as the preferential foreshortening of the molecule is altered, or the intersections of corresponding rel-rods in reciprocal space occur at different spatial frequencies.

Using a least squares, splined, fitting routine that uses Debye diffraction models for flat sheets, we compare the systematic differences that arise when fitting experimental data to those calculated for assemblages of randomly-oriented graphene, or faceted structures (Fig. 4) [5]. Visually, we see the similar inability of the flat sheet model to fit the amount of scattering at the (100) and (110) peaks in both the experimental data and the model ensemble of randomly-oriented graphene. The flat-sheet model also falls short on the leading edge of the graphene spacings in both cases. Thus, the inability of the flat sheet model to fit core electron diffraction may be due to coherence effects arising from inter-sheet relationships. 


\section{References:}

1. S. Amari, E. Zinner, and R.S. Lewis, Meteoritics 30, 679 (1995).

2. T. Bernatowicz, et. al., Astrophysical Journal 472, 760 (1996).

3. P. Fraundorf and M. Wackenhut, Ap. J. Lett. 578, L153 (2002).

4. B.E. Warren, Phys. Rev 59(9), 693 (1941).

5. B.E. Warren, X-Ray Diffraction, Addison-Wesley/Dover, New York (1969/1990).

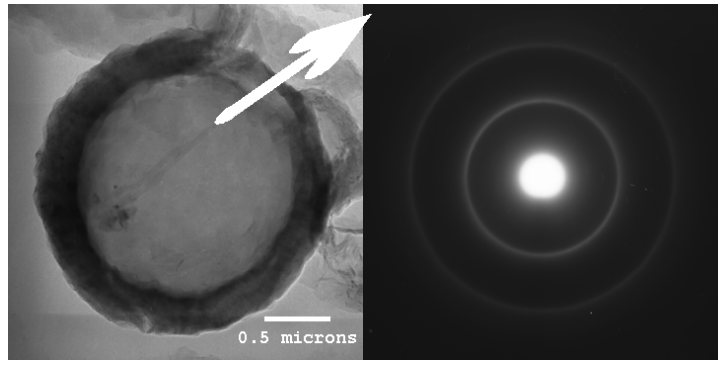

Figure 1: A presolar graphite spherule with a core-rim structure, and a diffraction pattern from the core. The (002) graphite spacing is absent in diffraction.
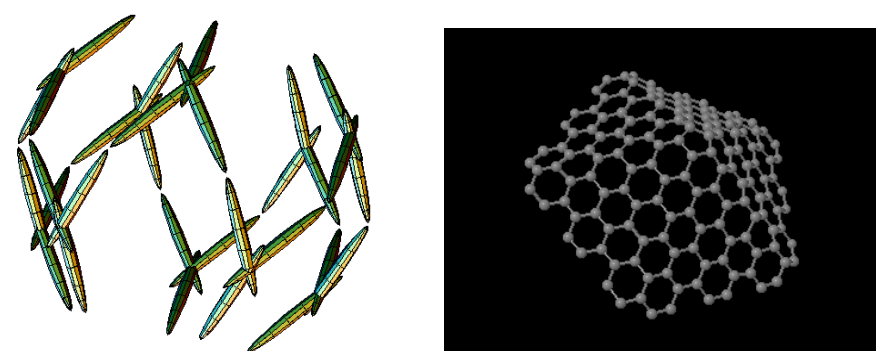

Figure 2: A picture of atom positions for a faceted carbon nanocone consisting of five flat faces, and the corresponding reciprocal lattice structure for (110) periodicity.

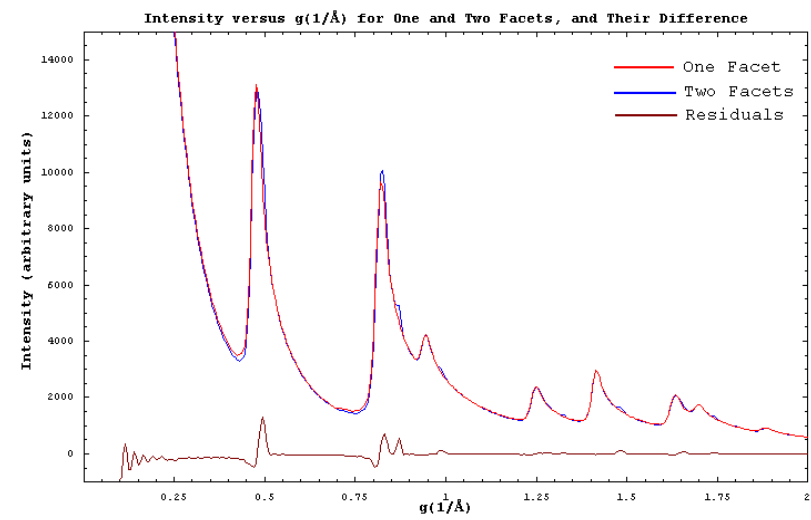

Figure 3: A comparison of Debye diffraction patterns for one and two facets of the faceted nanocone. Observe the occurrence of a satellite peak due to the orientation of the facets.
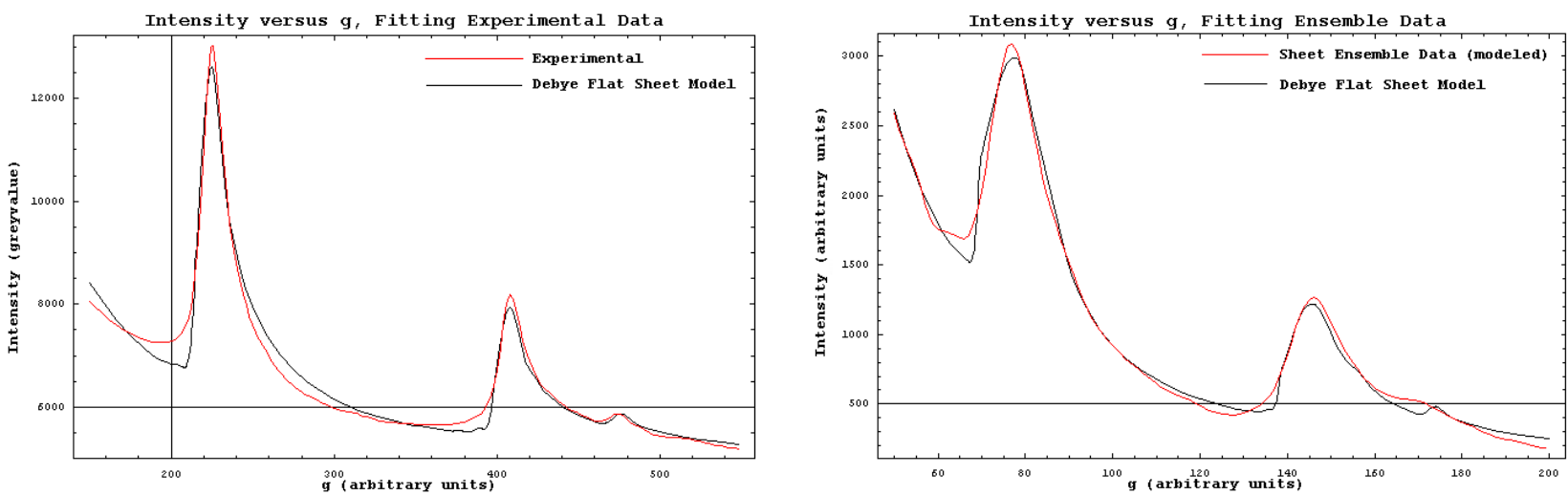

Figure 4: Debye flat sheet models fitted to experimental data and an ensemble of randomly-oriented flat sheets. Similarities in differences may arise, simply from coherence effects. 